\title{
Effective Hamiltonians for fastly driven tight-binding chains
}

\author{
A.P. Itin $^{1,2}$ and A.I. Neishtadt ${ }^{2,3}$ \\ ${ }^{1}$ Zentrum für optische Quantentechnologien, Universität Hamburg, \\ Luruper Chaussee 149, 22761 Hamburg, Germany \\ ${ }^{2}$ Space Research Institute, formerly Russian Academy of Sciences, Moscow, Russia \\ ${ }^{3}$ Department of Mathematical Sciences, Loughborough University, Loughborough, LE11 3TU, UK.
}

We consider a single particle tunnelling in a tight-binding model with nearest-neighbour couplings, in the presence of a periodic high-frequency force. An effective Hamiltonian for the particle is derived using an averaging method resembling classical canonical perturbation theory. Three cases are considered: uniform lattice with periodic and open boundary conditions, and lattice with a parabolic potential. We find that in the latter case, interplay of the potential and driving leads to appearence of the effective next-nearest neighbour couplings. In the uniform case with periodic boundary conditions the second- and third-order corrections to the averaged Hamiltonian are completely absent, while in the case with open boundary conditions they have a very simple form, found before in some particular cases by S.Longhi [Phys. Rev. B 77, 195326 (2008)]. These general results may found applications in designing effective Hamiltonian models in experiments with ultracold atoms in optical lattices, e.g. for simulating solid-state phenomena.

\section{INTRODUCTION}

Effective Hamiltonians created by high-frequency perturbations have many interesting applications in physics; a well-known counterintuitive example of induced effective potential is provided by Kapitza pendulum [1, 2]. We are interested in applying averaging methods of classical Hamiltonian mechanics (see, e.g., [3]) to quantum tight-binding models, which often arise in solid-state and, more generally, condensed-matter physics.

In solid-state physics, unusual transport phenomena may arise when an ac electric field is applied to the system, e.g. coherent desctruction of tunnelling and dynamic localization [4, 5]. Corresponding applications to coherent control of tunnelling and electronic transport in semiconductor superlattices and arrays of coupled quantum dots have been receiving a lot of interest lately [6, 7]. Very recently, in many experiments with atoms in optical lattices, effective Hamiltonians were created using high-frequency perturbations [5, 8, 9]. A particle in a deep optical lattice potential can be described by a tight-binding model. Applying a high-frequency force, one can engineer effective tunnelling constants in the model, which can be useful to mimic certain solid-state phe- 
nomena [8]. For many realistic applications of such type, it is important to derive accurate effective Hamiltonians taking into account higher-order terms [10]. Here we find a useful method for such derivation in the spirit of canonical perturbation theory, and apply it for several tight-binding systems. Our approach is based on idea of canonical transformations removing time-dependence from the Hamiltonian, which in the present context means unitary transformations of square matrices. Similar ideas have been applied to transport in classical periodic potentials [11 13]. In the next Section, the general method is outlined. In Section III, it is applied to three different tight-binding models.Our approach is actually not limited to tight-binding systems, but it becomes especially transparent and elegant for such kind of systems. Section IV gives concluding remarks.

\section{THE AVERAGING METHOD}

Consider a tight-binding model with the Hamiltonian

$$
H=J \sum(|n\rangle\langle n+1|+| n+1\rangle\langle n|)+\sum_{n} V(n)|n\rangle\left\langle n\left|+e d E(\omega t) \sum_{n} n\right| n\right\rangle\langle n|,
$$

where $J$ is the hopping parameter, $V(n)$ is the external potential (we consider only parabolic potential in this paper, $\left.V(n)=V \frac{n^{2}}{2}\right), d$ is the intersite distance, $E$ is the applied electric field, $e$ is the charge of the particle. The same model can be realized also with neutral particles, by approapriate shaking of the lattice.

Expanding a quantum state as $|\psi(t)\rangle=\sum c_{n}|n\rangle$, one gets a system of equations

$$
i \dot{c}_{n}=J\left(c_{n+1}+c_{n-1}\right)+V(n) c_{n}+\mathcal{E}(\omega t) n c_{n}
$$

It is convenient to make a trasformation $c_{n}(t)=x_{n}(t) \exp \left[-i n \int_{0}^{t} \mathcal{E}\left(t^{\prime}\right) d t^{\prime}\right]$, so that equations of motion are

$$
i \dot{x}_{n}=J\left(x_{n+1} F(t)+x_{n-1} F^{*}(t)\right)+V(n) x_{n},
$$

where $F(t)=\exp \left[-i \int_{0}^{t} \mathcal{E}\left(t^{\prime}\right) d t^{\prime}\right]=F_{0}+\sum F_{l} \exp (-i l \omega t), \mathcal{E}=e d E$.

Introducing fast time $t^{\prime}=\omega t \equiv t / \epsilon$, we get, in the matrix form,

$$
i \dot{X}=\epsilon H X
$$


where

$$
H=J\left(\begin{array}{cccc}
0 & F & \cdots & 0 \\
F^{*} & 0 & \ddots & \vdots \\
\vdots & \ddots & \ddots & F \\
0 & \cdots & F^{*} & 0
\end{array}\right) \equiv J\left(F \mathcal{U}+F^{*} \mathcal{B}\right)
$$

where $\mathcal{U}, \mathcal{B}$ are matrices with unities on the first upper- and lower- codiagonals, correspodingly $\left(\mathcal{U}_{m n}=\delta_{m, n+1}, \mathcal{B}_{m n}=\delta_{m, n-1}\right)$.

Secondly, consider the case of the chain with periodic boundary conditions, with the Hamiltonian

$$
H_{p}=J\left(\begin{array}{ccccc}
0 & F & 0 & \cdots & F^{*} \\
F^{*} & 0 & \ddots & \ddots & \vdots \\
\vdots & \ddots & \ddots & \ddots & 0 \\
0 & 0 & \ddots & 0 & F \\
F & 0 & \cdots & F^{*} & 0
\end{array}\right)
$$

Thirdly, in the case of a lattice with additional parabolic potential $\left(V(n)=V \frac{n^{2}}{2}\right)$ often employed in applications with ultracold atoms, the Hamiltonian is

$$
H_{p p}=J\left(\begin{array}{ccccc}
\frac{N^{2} V}{2 J} & F & 0 & \cdots & 0 \\
F^{*} & \frac{(N-1)^{2} V}{2 J} & \ddots & \ddots & \vdots \\
\vdots & \ddots & \ddots & \ddots & 0 \\
0 & 0 & \ddots & \frac{(N-1)^{2} V}{2 J} & F \\
0 & 0 & \cdots & F^{*} & \frac{N^{2} V}{2 J}
\end{array}\right)
$$

where $V$ is the strength of the parabolic potential, and the lattice has $(2 \mathrm{~N}+1)$ sites.

In the spirit of the Hamiltonian averaging method in classical mechanics, we are making a unitary transformation $X=C \tilde{X}$ so that equations for the transformed variables are

$$
i \dot{\tilde{X}}=\left[C^{-1} \epsilon H C-i C^{-1} \dot{C}\right] \tilde{X}
$$

We are looking for a transformation of the form $C=\exp \left[\epsilon K_{1}+\epsilon^{2} K_{2}+\epsilon^{3} K_{3}\right]$, where $K_{i}$ are skew-Hermitian time-periodic matrices, which would remove time-dependent terms from the Hamiltonian, leaving only time-independent terms. 
Generally, we have

$$
\begin{aligned}
C & \approx I+\epsilon K_{1}+\epsilon^{2}\left(\frac{1}{2} K_{1}^{2}+K_{2}\right)+\epsilon^{3}\left(\frac{1}{6} K_{1}^{3}+\frac{1}{2}\left(K_{1} K_{2}+K_{2} K_{1}\right)+K_{3}\right), \\
C^{\dagger} & \approx I-\epsilon K_{1}+\epsilon^{2}\left(\frac{1}{2} K_{1}^{2}-K_{2}\right)+\epsilon^{3}\left(-\frac{1}{6} K_{1}^{3}+\frac{1}{2}\left(K_{1} K_{2}+K_{2} K_{1}\right)-K_{3}\right),
\end{aligned}
$$

where $I$ is the unity matrix.

In the first order, we have

$$
i \dot{K}_{1}=H(t)-\langle H(t)\rangle \equiv\{H\}
$$

and therefore $i K_{1}=\int(H-\langle H\rangle) d t=\int\{H\} d t$. We introduce here curly brackets as taking timeperiodic part of a time-dependent function: $\{X\} \equiv X-\langle X(t)\rangle$, where $\langle X(t)\rangle \equiv \frac{1}{2 \pi} \int_{0}^{2 \pi} X\left(t^{\prime}\right) d t^{\prime}$.

In the second order,

$$
i \dot{K}_{2}=\left\{H K_{1}-K_{1} H-\frac{i}{2}\left(\dot{K}_{1} K_{1}-K_{1} \dot{K}_{1}\right)\right\}
$$

In the third order, we finally get

$$
\epsilon H_{e f f}=\epsilon H_{1}+\epsilon^{2} H_{2}+\epsilon^{3} H_{3},
$$

where

$$
\begin{aligned}
H_{1} & =\langle H\rangle \\
H_{2} & =\left\langle H K_{1}-K_{1} H-\frac{i}{2}\left(\dot{K}_{1} K_{1}-K_{1} \dot{K}_{1}\right)\right\rangle \\
H_{3} & =\left\langle H K_{2}-K_{2} H+\frac{1}{2}\left(H K_{1}^{2}+K_{1}^{2} H\right)-K_{1} H K_{1}-\frac{i}{2}\left(\dot{K}_{1} K_{2}-K_{1} \dot{K}_{2}+\dot{K}_{2} K_{1}-K_{2} \dot{K}_{1}\right)\right. \\
& \left.-\frac{i}{6}\left(\dot{K}_{1} K_{1}^{2}+K_{1}^{2} \dot{K}_{1}-2 K_{1} \dot{K}_{1} K_{1}\right)\right\rangle
\end{aligned}
$$

These general formulas can be applied to particular models, as done in the next Section.

One can also write expressions Eq.(13) in a more compact way:

$$
\begin{aligned}
& H_{1}=\langle H\rangle \\
& H_{2}=\frac{1}{2}\left\langle\left[\{H\}, K_{1}\right]\right\rangle \\
& H_{3}=\left\langle\left[H, K_{2}\right]+\frac{1}{2}\left[\left[H, K_{1}\right], K_{1}\right]-\frac{i}{2}\left(\left[\dot{K}_{1}, K_{2}\right]+\left[\dot{K}_{2}, K_{1}\right]\right)-\frac{i}{6}\left[\left[\dot{K}_{1}, K_{1}\right], K_{1}\right]\right\rangle,
\end{aligned}
$$


where square brackets denote matrix commutation: $[A, B]=A B-B A$.

\section{APPLICATIONS TO PARTICULAR MODELS}

For the uniform model with periodic boundary conditions (6), we get a very interesting and important result: $H_{2}=H_{3}=0$. First- and second-order corrections are completely absent in this case (note that, since the Hamiltonian $\epsilon H_{1}$ contains $\epsilon, H_{2}$ and $H_{3}$ define the first and the second-order corrections, correspondingly).

For the uniform model with open boundary conditions (5), we have

$$
\begin{gathered}
K_{1}=-i \int\{H\} d t=J\left(\begin{array}{cccc}
0 & L & \cdots & 0 \\
-L^{*} & 0 & \ddots & \vdots \\
\vdots & \ddots & \ddots & L \\
0 & \cdots & -L^{*} & 0
\end{array}\right)=J\left(L \mathcal{U}-L^{*} \mathcal{B}\right), \quad L \equiv \sum_{l \neq 0} \frac{F_{l}}{l} \exp (-i l t) \\
\dot{K}_{1}=-i\{H\}=-i J\left(\begin{array}{cccc}
0 & \tilde{F} & \cdots & 0 \\
\tilde{F}^{*} & 0 & \ddots & \vdots \\
\vdots & \ddots & \ddots & \tilde{F} \\
0 & \cdots & \tilde{F}^{*} & 0
\end{array}\right)=-i J\left(\tilde{F} \mathcal{U}+\tilde{F}^{*} \mathcal{B}\right), \quad \tilde{F} \equiv\{F\}=\sum_{l \neq 0} F_{l} \exp (-i l t) \\
H K_{1}-K_{1} H=J^{2}\left(\begin{array}{cccc}
-P & 0 & \cdots & 0 \\
0 & 0 & \ddots & \vdots \\
\vdots & \ddots & \ddots & 0 \\
0 & \cdots & 0 & P
\end{array}\right) \equiv-J^{2} P \mathcal{Z}_{1}, \quad P \equiv L F^{*}+F L^{*},
\end{gathered}
$$

where $\mathcal{Z}_{1}$ is a square matrix with $1,-1$ in the upper left and the bottom right corners, and zeros elsewhere.

$$
\begin{gathered}
\dot{K}_{1} K_{1}-K_{1} \dot{K}_{1}=i J^{2} D \mathcal{Z}_{1} \quad D \equiv L \tilde{F}^{*}+\tilde{F} L^{*} \\
K_{2}=-i J^{2} \mathcal{Z}_{1} T, \quad T \equiv \int\left\{-P+\frac{D}{2}\right\} d t \equiv \int S d t \\
\dot{K}_{2}=-i J^{2} \mathcal{Z}_{1} S
\end{gathered}
$$




$$
H K_{2}-K_{2} H=i T J^{3}\left(\begin{array}{ccccc}
0 & F & 0 & \cdots & 0 \\
-F^{*} & 0 & \ddots & \ddots & \vdots \\
\vdots & \ddots & \ddots & \ddots & 0 \\
0 & 0 & \ddots & 0 & F \\
0 & 0 & \cdots & -F^{*} & 0
\end{array}\right)=i T J^{3}\left(F \mathcal{U}_{1}-F^{*} \mathcal{B}_{1}\right)
$$

where $\mathcal{U}_{1}, \mathcal{B}_{1}$ are matrices with only two non-zero entries '1' on the ends of the upper- and lowerco-diagonal.

$$
\begin{gathered}
\frac{1}{2}\left(H K_{1}^{2}+K_{1}^{2} H\right)-K_{1} H K_{1}=-\frac{P J^{3}}{2}\left(L \mathcal{U}_{1}+L^{*} \mathcal{B}_{1}\right) \\
\frac{i}{6}\left(\dot{K}_{1} K_{1}^{2}+K_{1}^{2} \dot{K}_{1}-2 K_{1} \dot{K}_{1} K_{1}\right)=-\frac{D J^{3}}{6}\left(L \mathcal{U}_{1}+L^{*} \mathcal{B}_{1}\right) \\
\frac{i}{2}\left(\dot{K}_{1} K_{2}-K_{1} \dot{K}_{2}+\dot{K}_{2} K_{1}-K_{2} \dot{K}_{1}\right)=\frac{J^{3}}{2}\left(S\left[L \mathcal{U}_{1}+L^{*} \mathcal{B}_{1}\right]+i T\left[\tilde{F} \mathcal{U}_{1}-\tilde{F}^{*} \mathcal{B}_{1}\right]\right) .
\end{gathered}
$$

One obtains

$$
\begin{gathered}
\langle P\rangle=\langle D\rangle=2 \sum_{l=1} \frac{\left|F_{l}\right|^{2}-\left|F_{-l}\right|^{2}}{l} \equiv 2 D_{2} . \\
\langle L D\rangle=\sum_{k \neq 0} \sum_{l \neq 0}\left(\frac{F_{k} F_{l} F_{k+l}^{*}+F_{k} F_{l}^{*} F_{l-k}}{k l}\right) \equiv L_{3}, \\
\langle T F\rangle=\frac{i}{2} L_{3} .
\end{gathered}
$$

To conclude, in the case of open boundary conditions effective Hamiltonians have a very simple form

$$
H_{2}=J^{2} D_{2} \mathcal{Z}_{1}, \quad H_{3}=-\frac{J^{3}}{3}\left(L_{3} \mathcal{U}_{1}+L_{3}^{*} \mathcal{B}_{1}\right)
$$


Thirdly, in the model with parabolic potential (7) we have

$$
\begin{gathered}
K_{1}=J\left(L \mathcal{U}-L^{*} \mathcal{B}\right), \quad \dot{K}_{1}=J\left(\tilde{F} \mathcal{U}+\tilde{F}^{*} \mathcal{B}\right), \\
\dot{K}_{1} K_{1}-K_{1} \dot{K}_{1}=i J^{2} D \mathcal{Z}_{1} \\
H K_{1}-K_{1} H=J^{2}\left(\begin{array}{ccccc}
-P & \frac{2 N-1}{2} \frac{V}{J} L & 0 & \ldots & 0 \\
\frac{2 N-1}{2} \frac{V}{J} L^{*} & 0 & \frac{2 N-3}{2} \frac{V}{J} L & 0 & \vdots \\
\vdots & \ddots & \ddots & \ddots & 0 \\
0 & 0 & \ddots & 0 & -\frac{2 N-1}{2} \frac{V}{J} L \\
0 & 0 & \cdots & -\frac{2 N-1}{2} \frac{V}{J} L^{*} & P
\end{array}\right)
\end{gathered}
$$

The first correction to the averaged Hamiltonian $\epsilon^{2} H_{2}$ looks exactly the same as that of the uniform case, and does not depend on the potential. In the following, we neglect influence of the boundary conditions, assuming the lattice is very long. Then, the first correction is absent, while the second correction $\epsilon^{3} H_{3}=\epsilon^{3} J^{2} V M$ contains contribution from the parabolic potential. $M$ is a 5-diagonal matrix, with the following entries (non-zero diagonals are listed from top to bottom, with $\left({ }^{\prime} 0^{\prime}\right)$ denoting the main diagonal, $\left({ }^{\prime}+2^{\prime}\right)$ and $\left({ }^{\prime}+1^{\prime}\right)$ upper co-diagonals, $\left({ }^{\prime}-2^{\prime}\right)$ and $\left({ }^{\prime}-1^{\prime}\right)$ lower co-diagonals) :

$$
\begin{aligned}
& \left({ }^{\prime}+2^{\prime}\right) \quad\left(i\left\langle F L_{2}\right\rangle-\frac{i}{2}\left\langle\tilde{F} L_{2}\right\rangle\right) \delta_{m, n+2} \\
& \left({ }^{\prime}+1^{\prime}\right) \quad 0 \\
& \left({ }^{\prime} 0^{\prime}\right) \quad\left(-i\left\langle F^{*} L_{2}+F L_{2}^{*}\right\rangle+\frac{i}{2}\left\langle\tilde{F}^{*} L_{2}+\tilde{F} L_{2}^{*}\right\rangle\right) \delta_{m, n} \\
& \left({ }^{\prime}-1^{\prime}\right) 0 \\
& \left({ }^{\prime}-2^{\prime}\right) \quad\left(i\left\langle F^{*} L_{2}^{*}\right\rangle-\frac{i}{2}\left\langle\tilde{F}^{*} L_{2}^{*}\right\rangle\right) \delta_{m, n-2}, \\
& L_{2}=i \sum_{l \neq 0} \frac{F_{l}}{l^{2}} \exp (-i l t),\left\langle L_{2}\right\rangle=0,\left\langle F L_{2}\right\rangle=\left\langle\tilde{F} L_{2}\right\rangle=i \sum_{l \neq 0} \frac{F_{l} F_{-l}}{l^{2}}
\end{aligned}
$$

One can see that this correction creates next-nearest-neighbour couplings: non-zero entries are not on the main co-diagonals, as it would be in case of nearest-neighbour couplings, but on the next-to main co-diagonals. Since $\left\langle F^{*} L_{2}+F L_{2}^{*}\right\rangle=\left\langle\left(\sum_{l \neq 0} F_{l}^{*} \exp (i l t) i \sum_{m \neq 0} \frac{F_{m}}{m^{2}} \exp (-i m t)\right)+\right.$ 
$\left.\left(\sum_{l \neq 0} F_{l} \exp (-i l t)(-i) \sum_{m \neq 0} \frac{F_{m}^{*}}{m^{2}} \exp (i m t)\right)\right\rangle=0$, finally the second correction has a very simple, twodiagonal form

$$
\begin{aligned}
\epsilon^{3} H_{3}^{m n} & =\epsilon^{3} J^{2} V \frac{i}{2}\left(\left\langle F L_{2}\right\rangle \delta_{m, n+2}+\left\langle F^{*} L_{2}^{*}\right\rangle \delta_{m, n-2}\right) \\
& =-\frac{\epsilon^{3}}{2} J^{2} V\left(\sum_{l \neq 0} \delta_{m, n+2} \frac{F_{l} F_{-l}}{l^{2}}+\delta_{m, n-2} \frac{F_{l}^{*} F_{-l}^{*}}{l^{2}}\right)
\end{aligned}
$$

Consider a particular case of harmonic driving, with $\mathcal{E}=\mathcal{E}_{0} \cos t$. We have $F_{l}=J_{l}\left(\mathcal{E}_{0}\right)$. The induced next-nearest neighbour coupling is $J^{\prime}=-\epsilon^{3} J^{2} V \sum_{l>0} \frac{(-1)^{l} J_{l}^{2}\left(\mathcal{E}_{0}\right)}{l^{2}}$

Returning from the fast time back to the original time, we have

$$
J^{\prime}=-\frac{J^{2} V}{\omega^{2}} \sum_{l>0} \frac{(-1)^{l} J_{l}^{2}\left(\mathcal{E}_{0}\right)}{l^{2}}
$$

As a function of $\mathcal{E}_{0}$, it has an oscillatory form, and one can choose parameters that nullify the next-neighbour coupling (e.g., $\mathcal{E}_{0}=3.32,4.11$, etc), or maximize it (e.g., $\mathcal{E}_{0}=1.77,5.24$, etc). It can be tuned to be either positive or negative, which may be useful for applications.

\section{CONCLUSIONS}

The approach based on canonical transformations and described in Section II has been applied to three different lattice systems: uniform lattice with open boundary conditions, uniform lattice with periodic boundary conditions, and a lattice with an additional parabolic potential. In the first case, we generalize results obtained by S. Longhi [10]. In particular, we show that second-order corrections have very simple ('boundary') form. In the second case, we get a very interesting and unexpected result: absence of corrections to the averaged Hamiltonian in the second and third order. In the case of external parabolic potential, another unexpected result is found: interplay of driving and non-uniform external potential creates effective (uniform!) next-nearest neighbour couplings. The same result can be obtained in the semiclassical approach [14]. These results, we believe, may found applications in forthcoming experiments with cold atoms in driven optical lattices.

This work was partially supported by RFBR (project no. 13-01-00251). A.P.I thanks A.Polkovnikov, M.Thorwart, A.Eckardt, A.Engel and L.Mathey for interesting and simulating 
discussions.

[1] N.N. Bogolyubov, The Theory of Perturbations in Nonlinear Mehanics, Proc. Inst. Struct. Mech., no. 14, 9 (1950) [In Russian: Teoriya vozmusheniy v nelineynoy mekhanike. Sbornik trudov Instituta stroitel'noy mekhaniki AN USSR, no. 14, 9 (1950) ].

[2] P.L. Kapitza, Dynamic stability of a pendulum when its point of suspension vibrates, Soviet Phys. JETP 21, 588 (1951).

[3] V. I. Arnold, V. V. Kozlov, and A. I. Neishtadt, Mathematical Aspects of Classical and Celestial Mechanics, 3rd ed. (Springer, Berlin, 2006).

[4] D.H.Dunlap and V.M. Kenkre, Phys. Rev. B 34, 3625 (1986).

[5] M. Holthaus, Phys. Rev. Lett. 69, 351 (1992).

[6] M. Holthaus, D.W. Hohe, Philos. Mag. B 74, 105 (1996).

[7] M. Glck, A.R. Kolovsky, and H.J. Korsch, Phys. Rep. 366 , 103 (2002).

[8] N. Strohmaier, Y. Takasu, K. Gunter, R. Jordens, M. Kohl, H. Moritz, and T. Esslinger, Phys. Rev. Lett. 99, 220601 (2007); A. Alberti et al., Nat. Phys. 5, 547 (2009); A. Zenesini et al., Phys. Rev. Lett. 102, 100403 (2009); A. Eckardt et al., Europhys. Lett. 89, 10010 (2010).

[9] K. Kudo, T. Boness, and T. S. Monteiro, Phys. Rev. A 80, 063409 (2009); K. Kudo and T. S. Monteiro, ibid. 83, 053627 (2011); A. R. Kolovsky, E. A. Gomez, and H. J. Korsch, ibid. 81, 025603 (2010).

[10] S. Longhi, Phys. Rev. B 77, 195326 (2008)

[11] A. P. Itin, R. de la Llave, A. I. Neishtadt, and A. A. Vasiliev, Chaos 12, 1043 (2002).

[12] X. Leoncini, A. I. Neishtadt, and A. A. Vasiliev, Phys. Rev. E 79, 026213 (2009).

[13] A. P. Itin and A. I. Neishtadt, Phys. Rev. E 86, 016206 (2012)

[14] A.P.Itin, L.Mathey, in preparation. 